NISTIR 7300

\title{
NIST FINGERPRINT IMAGE QUALITY (NFIQ) COMPLIANCE TEST
}

Elham Tabassi 
NISTIR 7300

\section{NIST FINGERPRINT IMAGE QUALITY (NFIQ) COMPLIANCE TEST}

Elham Tabassi

Image Group

Information Technology Laboratory

February 2005

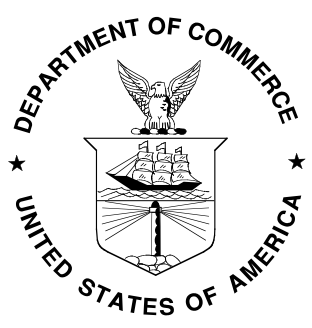

U.S. DEPARTMENT OF COMMERCE

Carlos M. Gutierrez, Secretary NATIONAL INSTITUTE OF STANDARDS AND TECHNOLOGY William Jeffery, Director 


\title{
NFIQ COMPLIANCE TEST \\ Elham Tabassi \\ National Institute of Standards and Technology \\ Gaithersburg, Maryland
}

\begin{abstract}
In August 2004 NIST published a novel measure of fingerprint image quality, which is designed to predict performance of minutia-based fingerprint matching systems. The definition and methodology of NIST Fingerprint Image Quality (NFIQ) is documented in [1] and its implementation is publicly available; but subject to US export control laws as part of NIST Fingerprint Image Software 2 (NFIS2) distribution [2]. This document provides a self administered compliance test that tests if an installation of NFIQ from NFIS2 distribution produces the same results as the original implementation of NFIQ that was developed, tested and published by NIST in August 2004.
\end{abstract}

\section{Introduction}

The term compliance describes the ability to act according to a set of rules or requests. In this case, we want to study the degree of similarity between any installations of NIST Fingerprint Image Quality (NFIQ) software from NFIS2 distribution [2] to the original implementation developed, tested, and published by NIST (original NIST NFIQ). Thereby we studied the NFIQ's sensitivity / variability when compiled and installed on different platforms. We compiled NFIQ from NFIS-2 CD-ROM on Windows XP ${ }^{1}$, Windows 2000, MAC OS X, and Red Hat Linux. We found the NFIQ numbers for 84,000 fingerprint images computed on each of the platforms listed above and the ones computed by the original implementation of NFIQ to be identical. That means NFIQ implementation is not sensitive to platforms. Therefore, we conclude that an installation of NFIQ, from NFIS2 CD-ROM, is compliant with the original NIST NFIQ implementation if the quality number assigned by both is the same. We provide a test program and a set of 1,145 images from Special Dataset 29 [3] with their original NIST NFIQ numbers to test compliance of an installation of NFIQ from NFIS2. NFIQ Compliance test suite can be downloaded from fingerprint.nist.gov/NFIS.

1 - Disclaimer - These procedures were developed for the Department of Justice in accordance with section 303 of the Border Security Act, codified as 8 U.S.C. 1732. Specific hardware and software products identified in this report were used in order to conduct the tests described in this document. In no case does such identification imply recommendation or endorsement by the National Institute of Standards and Technology, nor does it imply that the products and equipment identified are necessarily the best available for the purpose. 
This test has been designed to test the compliance of an installation of NFIQ from NFIS CD-ROM with the original NIST NFIQ, however, it is applicable to the cases that NFIQ source code is modified (for example for improved speed). If this compliance test fails for a modified version of NFIQ, NIST will consider testing the compliance of the modified version at NIST on a case-by-case basis.

The rest of this document is organized as follows. We detail robustness of NFIQ implementation to platforms in section 2. This precedes the main goal of this document in section 3, which discusses NFIQ compliance test procedures. Conclusions follow in section 4 .

\section{Testing NFIQ's sensitivity / variability to platforms}

The question arises that whether compiling NFIQ on platforms different than what it was developed originally will affect its outcome. The original NFIQ implementation was compiled on a Pentium IV Red Hat 7.2 Linux with gcc version 2.96. We compiled and installed NFIQ software from the NFIS2 CD-ROM [2] on several conventional platforms and compared the NFIQ numbers for 84,000 fingerprints of different fingers (right / left index and thumb), different impressions (rolled and flat), captured with different devices at different operational settings. In all cases, the quality numbers computed by the installation of NFIQ on different platforms were identical to the ones computed by the original NFIQ implementation. That means any installation of NFIQ from NFIS2 distribution on environments similar to ones tested shall produce same NFIQ numbers as the original NIST NFIQ implementation. A description of the platform test parameters follows.

\section{i. Compiler}

It is important to note that on all platforms tested, we compiled NFIQ using different versions of gcc, the GNU Compiler Collection, which can be downloaded for free from http://gcc.gnu.org. The original NFIQ implementation was compiled using gcc 2.96.

\section{ii. Operating Systems}

We compiled and installed NFIQ on Microsoft Windows 2000, and Microsoft Windows $\mathrm{XP}$ by first installing the free, publicly available Cygwin library and associated tools (www.cygwin.com). We also compiled and installed NFIQ on Mac OS X, and Red Hat 8.0 Linux. The original NFIQ's platform was Red Hat 7.2 Linux.

\section{iii. Hardware}

Machines used for this test had Intel Pentium III, Mobile Pentium IV, Intel Xenon, and PowerPC G4 processors. The original NFIQ was developed on a Pentium IV machine. Table 1 summarizes the platform parameters. The last row shows the platform used at NIST to develop NFIQ. 


\begin{tabular}{||l|l|l|l||}
\hline \hline OS PLATFORM & ARCHITECTURE & $\begin{array}{l}\text { MANUFACTUR } \\
\text { E/MODEL }\end{array}$ & COMPILER \\
\hline Windows XP & Mobile Pentium 4 & Toshiba & gcc 3.4 .5 \\
\hline Windows 2000 & Pentium III & Dell OptiPlex & gcc 3.3.3 \\
\hline Mac OS 10.3.6 & Macintosh PowerBook G4 & PowerPc G4 & gcc 3.3 \\
\hline Red Hat Linux 8.0 & Xenon & Dell precision & gcc 3.2 \\
\hline Red Hat Linux 7.2 & Pentium IV & Dell & gcc 2.96 \\
\hline \hline
\end{tabular}

Table 1. Platforms, and compilers used to test NFIQ robustness. The last row (in red) shows the platform used at NIST to develop NFIQ.

iv. Data

Fingerprint images from TXDPS and VISIT_POE data sets of SDK tests [4] were used to test NFIQ's sensitivity / variability to platforms. As it is shown in Table 2, this data is a reasonable mixture of different finger positions, and impression types. It is important to note that these data are for internal NIST testing only because it is sensitive but unclassified (SBU) government use only and cannot be publicly released.

For all the platforms listed in Table1, NFIQ numbers computed for the 84,000 fingerprints listed in Table 2 were compared with NFIQ numbers computed by the original NIST NFIQ implementation. In all cases, the computed NFIQ numbers were identical to the original NIST NFIQ implementation. Given the size and diversity of data in Table 2, we conclude that any installation of NIST Fingerprint Image Quality Software compiled with gcc on the platforms listed in Table 2 shall result in the same NFIQ number for a given image. Therefore, an installation of NFIQ Software, from NFIS2 CD-ROM, is compliant with the original NIST implementation of NFIQ if the quality number assigned by both is identical. To that end, we are providing a selfadministered NFIQ compliance test suit to test compliance of any installation of NFIQ to the original NIST NFIQ implementation.

\section{NFIQ Compliance Test Suite}

The main purpose of this document is provide tools to check if an installed version of NIFQ from NFIS2 CD-ROM produces the same results as the original NIST NFIQ implementation. As discussed in the previous section, we extensively tested NFIQ's sensitivity to other platforms and found it quite robust to the environments tested. That means a proper installation of NFIQ shall produce exact same results as the original NIST NFIQ implementation. NFIQ compliance test suite (NFIQ_Compliance_Test_Suite.tar) is provided to check the compliance of an installation of NFIQ from the NFIS2 distribution to the original NIST implementation. It is a self-administered test, which can be downloaded from fingerprint.nist.gov/NFIS. It contains a test program, and a set of fingerprint images in ANSI/NIST format from 
SD29 along with their original NFIQ numbers for comparison. An installation of NFIQ

\begin{tabular}{||l|l|l|l||}
\hline DATA SET & SIZE & IMPRESSION & $\begin{array}{l}\text { FINGER } \\
\text { POSITION }\end{array}$ \\
\hline TXDPS & 6000 & Rolled - inked & Right thumb \\
\hline TXDPS & 6000 & Rolled - inked & Left thumb \\
\hline TXDPS & 6000 & Rolled - inked & Right index \\
\hline TXDPS & 6000 & Rolled - inked & Left index \\
\hline TXDPS & 6000 & Plain - inked - segmented from slap & Right thumb \\
\hline TXDPS & 6000 & Plain - inked segmented from slap & Left thumb \\
\hline TXDPS & 6000 & Plain - inked segmented from slap & Right index \\
\hline TXDPS & 6000 & Plain - inked segmented from slap & Left index \\
\hline VISIT_POE & 18000 & Plain - live scan & Right index \\
\hline VISIT_POE & 18000 & Plain - live scan & Left index \\
\hline \hline
\end{tabular}

Table 2. Description of platforms test data

is compliant with the original NIST NFIQ implementation if it computes identical quality numbers for images in the compliance_testset with the original NIST NFIQ numbers that are provided as part of the test suite.

An explanation of NFIQ Compliance Test Suite components follows.

1. NFIQ_compliance_testset

This directory contains 1,145 images from SD29 [3], which are provided for compliance test. There are 392 rolled and 753 flat impressions of right/left thumb/index/middle fingers.

2. compliance_testset_nfiq.txt

This file contains the NFIQ numbers for images in the NFIQ_compliance_testset directory, which are computed by the original NFIQ implementation. We refer to these as the original NFIQ numbers.

\section{3. run_compliance_test.csh}

Script to test compliance. It gets the full path to the local NFIQ installation (binary to be tested) as input. The script computes NFIQ numbers for the images in the NFIQ_compliance_testset, and generates the file my_nfiq_numbers.txt. It then checks for any differences between the computed NFIQ numbers 
(my_nfiq_numbers.txt) and the original NFIQ numbers in compliance_testset_nfiq.txt and reports whether or not the two are identical.

It also computes and prints a cross-tabulation of occurrence frequencies of the original and computed NFIQ numbers. Each column of the matrix represents the instances in my_nfiq_numbers.txt (i.e. the computed NFIQ numbers), while each row represents the instances in compliance_testset_nfiq.txt (i.e. original NFIQ numbers). For compliance, all off-diagonal elements shall be zero. The benefit of this crosstabulation is that it is easy to examine the cases that an installation of NFIQ mislabels one class of NFIQ as another.

To run NFIQ_Compliance_Test_Suite, first have to untar it:

$$
>\text { tar-xvf NFIQ_Compliance_Test_Suite.tar }
$$

Then run the script

$>$./run_compliance_test.csh <path_to_local_NFIQ(binary) $>$

Figure 1. shows a successful compliance test.

The main focus in designing this compliance test was to test the compliance of an installation of NFIQ from NFIS CD-ROM, but this test is also applicable to cases where NFIQ source code has been modified, for example to improve speed. However, satisfying absolute agreement between a modified version of NFIQ and original NFIQ (diagonal confusion matrix of Figure 1) might be overly limiting, and some small degree of tolerance should be allowed, i.e. instead of absolute agreement, a confidence interval should be specified on the diagonal elements of the confusion matrix of Figure 1. Since our tests (see section II) did not show any variations, we cannot estimate the confidence intervals. However, if this compliance test fails for a modified version of NFIQ, NIST will consider testing the compliance of the modified version at NIST on a case-by-case basis. Interested parties can contact the author to further discuss the possibility of testing the compliance of their modified version of NFIQ at NIST.

\section{Conclusion}

We have addressed NFIQ's robustness to other platforms. We have provided a selfadministered NFIQ Compliance Test Suite that test if an installation of NFIQ from NFIS2 distribution is compliant with the original implementation of NFIQ that was developed, tested and published by NIST in August 2004 (original NIST NFIQ). The test suite contains a test program, and test data along with their original NFIQ numbers for comparison. An installation of NFIQ is compliant with the original NIST NFIQ implementation if for all images in the test data set, it computes identical NFIQ numbers with the original NIST NFIQ numbers. NFIQ Compliance Test Suite can be downloaded from fingerprint.nist.gov/NFIS. For cases that this compliance test fails due to modification in NFIQ source code, the author should be contacted for possible compliance test at NIST. 


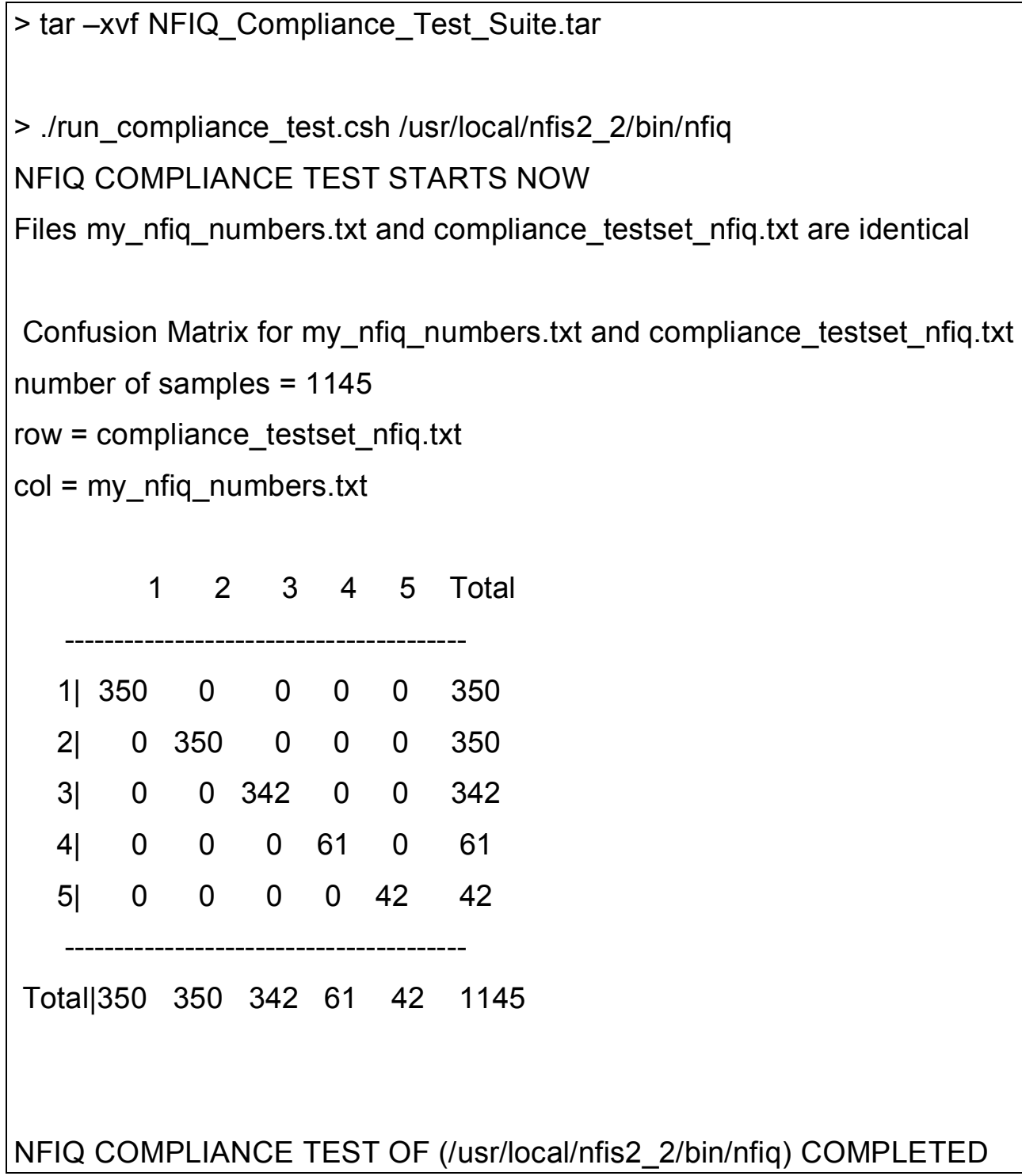

Figure 1. A successful NFIQ compliance test

\section{References}

[1] E. Tabassi, C. L. Wilson, C. Watson. NIST Fingerprint Image. Quality NISTIR 7151.

[2] M.D. Garris, C.I. Watson, R.M. McCabe, C.L. Wilson. User's guide to NIST fingerprint image software. NISTIR 6813. http://fingerprint.nist.gov/NFIS/index.html

[3] C. Watson. NIST Special Database 29 Plain and Rolled Images from Paired 
Fingerprint Cards, NIST, November 2001. http://www.nist.gov/srd/nistsd29.htm

[4] C. Watson, A. Wilson, K. Marshall, M. Indovina, R. Snelick. Studies of one-to-one fingerprint matching with vendor SDK matchers. NISTIR 7119

\section{Acronyms}

NFIS2 NIST Fingerprint Image Software 2

NFIQ NIST Fingerprint Image Quality

SD29 Special Dataset 29

Original NIST NFIQ The original implementation of NFIQ developed, tested, and published by NIST in August 2004 